\title{
Epidemiology and treatment patterns for locally advanced or metastatic urothelial carcinoma: a systematic literature review and gap analysis
}

Zsolt Hepp, PharmD, MS; Sonali N Shah, RPH, MBA, MPH; Karen Smoyer, PhD; and Pratyusha Vadagam, MSc

\section{What is already known about this subject}

- Several immuno-oncology (IO) agents targeting programmed death-1/deathligand 1 (PD-1/L1) are now approved second-line treatment options in the United States and Europe for patients with locally advanced or metastatic urothelial carcinoma $(\mathrm{la} / \mathrm{mUC})$ previously treated with platinum-based chemotherapy or first-line options in patients ineligible for cisplatin whose tumors express PD-L1 or for any platinum-based chemotherapy regardless of PD-L1 expression levels.

- There is a need to summarize the current literature on the epidemiology of la/mUC and the realworld treatment patterns since the introduction of these novel therapies to have a better understanding of what has already been published and identify evidence gaps for future research.

\author{
What this study adds \\ - The study found that there is a \\ lack of up-to-date, country-specific \\ epidemiological data on urothelial \\ carcinoma (UC) and la/mUC, including \\ rates of progression from early-stage \\ disease to la/mUC. \\ - There was a large variation in the rates \\ of treatment, particularly with cisplatin, \\ both within and among countries. \\ - The few published real-world IO studies \\ indicate there has been an increase \\ in the use of 10 therapy for la/mUC in \\ recent years; however, relatively high \\ levels of treatment discontinuation \\ were reported in patients treated with \\ 10 , and only a small percentage of \\ patients received subsequent therapy.
}

literature on the epidemiology of $\mathrm{la} / \mathrm{mUC}$ is limited, and real-world treatment patterns are not well established, especially with respect to therapies used following IO. (IO) agents targeting programmed death-1 or programmed death-ligand 1 (PD-1/L1) are approved second-line therapy options for patients with locally advanced or metastatic urothelial carcinoma (la/mUC) previously treated with platinum-based chemotherapy or first-line options in patients ineligible for cisplatin whose tumors express PD-L1 or for any platinum-based chemotherapy regardless of PD-L1 expression levels. However,
OBJECTIVES: To (a) report the epidemiology of urothelial carcinoma (UC) and la/mUC; (b) identify and summarize the published literature on la/mUC treatment patterns, including $\mathrm{IO}$ and post-IO treatment; and (c) identify evidence gaps.

METHODS: A systematic literature review was conducted using Cochrane dual-reviewer

\section{Author affiliations \\ Zsolt Hepp, PharmD, MS, Seagen, Bothell, WA, and Sonali N Shah, RPH, MBA, MPH, Astellas Pharma, Northbrook, IL. Karen Smoyer, PhD, and Pratyusha Vadagam, MSc, Envision Pharma Group, Philadelphia, PA.}

AUTHOR CORRESPONDENCE:

Zsolt Hepp, 425.527.2561;

zhepp@seagen.com

J Manag Care Spec Pharm 2021;27(2):240-55

Copyright $(2021$, Academy of Managed Care Pharmacy. All rights reserved.

methodology and the Preferred Reporting Items for Systematic Review and MetaAnalysis Protocols. Literature databases and selected congress abstracts (2017-2018) were searched for retrospective studies published January 2013-August 2018 in English reporting epidemiological and treatment data (all lines of therapy) for adult patients with la/mUC.

RESULTS: Among 6,584 database references and 1,832 congress abstracts screened, 45 publications (29 manuscripts, 1 poster, 15 abstracts; reporting 37 unique studies) were retained. All studies related to 
treatment patterns, and the majority were from the United States $(n=17)$, Japan $(n=8)$, and the United Kingdom $(n=5)$. Epidemiological data were not identified among the searches thus online registries were leveraged. Among the identified publications, 21 (20 unique) reported on cisplatin versus non-cisplatin regimens, 14 (8 unique) on 10 , and 9 ( 7 unique) on vinflunine. Cisplatin use varied both within and among countries (ranging from $18.4 \%$ in 1 U.S. study to $87.9 \%$ in 1 Japanese study). The use of 10 was higher in later lines of therapy, ranging from $1.4 \%$ to $7.9 \%$ as first-line therapy to $57.8 \%$ as second-line and $64.4 \%$ as third-line therapy. Among studies reporting 10 discontinuation rates, $41.4 \%-71 \%$ of patients were reported to discontinue 10 across the studies, and the median time to discontinuation ranged from 2.7 to 5.8 months. Only $25 \%-35.5 \%$ of patients received subsequent therapy following 10 discontinuation; post-IO treatments varied widely.

CONCLUSIONS: Additional published data on the country-specific epidemiology of UC and la/mUC are needed, including rates of progression from early-stage disease to la/mUC. There was large variation in treatment rates, particularly cisplatin use, within and across countries. The few published real-world IO studies reported high levels of discontinuation with only a small percentage of patients receiving subsequent therapy. As IO therapies continue to be granted regulatory approval in countries outside the United States and novel therapies gain approval in the post-IO setting, the treatment paradigm for patients with la/mUC is shifting, and future studies with more recent data will be required.

Bladder cancer is the most common malignancy in the urinary system with an estimated 549,393 new cases and 199,922 deaths worldwide in 2018. ${ }^{1}$ Urothelial carcinoma (UC) is the most common histological form of bladder cancer and accounts for $>90 \%$ of all cases. ${ }^{2,3}$ Although the majority of UC cases are superficial, an estimated $25 \%$ are muscle invasive, ${ }^{4}$ and $11 \%$ are locally advanced or metastatic (la/mUC) at diagnosis. ${ }^{5}$ Prognosis is poor for patients with la/mUC and $<15 \%$ survive beyond 5 years ${ }^{6}$; metastatic disease is considered incurable, and the 5 -year survival rate is $4.6 \%$ in the United States. ${ }^{5}$

Cisplatin-containing combination chemotherapy remains the standard of care for the first-line treatment of patients with la/mUC. ${ }^{7}$ However, approximately $30 \%-50 \%$ of patients are unfit for cisplatin-containing chemotherapy due to an Eastern Cooperative Oncology Group performance status $\geq 2$, creatinine clearance $<60 \mathrm{~mL} / \mathrm{min}$, presence of significant hearing loss or peripheral neuropathy, or heart failure. ${ }^{8,9}$ In patients for whom cisplatin-containing chemotherapy is unsuitable, carboplatin-based therapy may be offered as alternative first-line treatment. ${ }^{10}$ Overall survival ranges between 9 and 15 months in patients with la/mUC treated with first-line platinum-based chemotherapy.,

In recent years, several immuno-oncology (IO) agents targeting programmed death-1 or programmed death-ligand 1 (PD-1/L1) have gained full or accelerated approval in multiple countries for patients with la/mUC (the first U.S. Food and Drug Administration [FDA] approval for atezolizumab was in May 2016, with other agents approved February-May 2017). ${ }^{11}$ Current National Comprehensive Cancer Network NCCN Clinical Practice Guidelines in Oncology (NCCN Guidelines) recommend the use of anti-PD-1/L1 therapy as preferred second-line options following platinum-based chemotherapy; atezolizumab and pembrolizumab are also recommended as preferred first-line options in patients ineligible for cisplatin-based chemotherapy whose tumors express PD-L1 or who are not eligible for any platinum-based chemotherapy regardless of PD-L1 expression levels. ${ }^{12,13}$ The European Society for Medical Oncology (ESMO) treatment guidelines recommend pembrolizumab in patients with la/mUC who progress after platinum-based chemotherapy; atezolizumab and nivolumab can be considered in this setting although they are supported by lower levels of evidence (avelumab and durvalumab have not received European Medicines Agency [EMA] approval in this setting). ${ }^{14}$ ESMO also recommends atezolizumab and pembrolizumab as first-line therapy options in cisplatin-ineligible patients with PD-L1-positive la/mUC. ${ }^{14}$ Although some patients with la/mUC may experience durable responses with antiPD-1/L1 therapy, data from clinical trials show objective response rates ranging from $23 \%$ to $29 \%$ as first-line therapy in unselected populations and from $13 \%$ to $21 \%$ as second-line therapy. ${ }^{15-17}$

For patients with la/mUC who are refractory to platinum-based chemotherapy and IO, treatment options have been limited (as of the time of publication). In December 2019, enfortumab vedotin-ejfv (EV) received FDA accelerated approval for adult patients with la/mUC who have previously received a PD-1/L1 inhibitor and a platinumbased chemotherapy in the neoadjuvant/adjuvant, la/mUC setting. ${ }^{18} \mathrm{EV}$ is now recognized as a recommended, preferred subsequent treatment option in this setting in the NCCN and ESMO guidelines. ${ }^{12,14}$ Erdafitinib, a fibroblast growth factor receptor (FGFR) inhibitor, although not specifically indicated for use in the post-IO setting, has FDA regulatory approval in the post-platinum setting for patients with la/mUC who harbor susceptible FGFR3 or FGFR2 genetic alterations and is a recommended and preferred option in this specific population in the NCCN and ESMO guidelines. ${ }^{12,14,19} \mathrm{EV}$ and erdafitinib are not approved outside the United States. Before these agents, vinflunine was approved by the EMA in 2009 for patients with la/mUC 
after failure of platinum-containing therapy and has been a treatment option specifically in Europe, although its use is now only recommended if IO or combination chemotherapy are unsuitable.,20 Vinflunine is not approved in the United States and not recommended in the United Kingdom. ${ }^{21}$

Given the relatively short time period since IOs were first approved for the treatment of la/mUC, there is limited literature on real-world treatment patterns after the introduction of these therapies, especially pertaining to novel therapies that may be used following discontinuation of IO. Although Cumberbatch et al. (2018) recently published a detailed review of the epidemiology of bladder cancer, it focused on the contemporary risk factors associated with the disease and did not cover treatment patterns. ${ }^{22}$ The present systematic literature review (SLR) was conducted to (a) report the epidemiology of UC and la/mUC; (b) identify and summarize the published literature on la/mUC treatment patterns, including the percentage of patients treated with cisplatin- versus non-cisplatin-based regimens, IO therapy (by line of therapy [LOT]), and subsequent drug therapy after receipt of IO therapy; and (c) identify gaps in the literature on epidemiology and treatment patterns and assess unmet needs post-IO.

\section{Methods}

\section{SEARCH STRATEGY}

The SLR was conducted using Cochrane dual-reviewer methodology and followed the Preferred Reporting Items for Systematic Reviews and Meta-Analysis Protocols (PRISMA-P) checklist. To capture contemporary data spanning the pre- and postregulatory approval of IO agents for $\mathrm{la} / \mathrm{mUC}$, searches were focused on studies published from January 1, 2013, to August 24, 2018, in MEDLINE with MEDLINE epubs ahead of print, Embase, and Cochrane via Ovid. Supplementary searches were performed in PubMed (past 2 years) using text word or Medical Subject Headings to identify retrospective studies published in English that reported epidemiology or treatment data for adult patients with UC or la/mUC.

In addition to the literature database searches, the reference section of identified review articles was reviewed for relevant studies. Abstracts presented at the following conferences held in 2017 and 2018 were also searched: International Society for Pharmacoeconomics and Outcomes Research, American Society of Clinical Oncology, American Urological Association, European Association of Urology, ESMO, and AMCP. Supplemental searches of recognized national and international epidemiology websites were conducted, including Surveillance, Epidemiology, and End Results; European Cancer Observatory; Cancer Research UK; U.K. Office for National Statistics; European Cancer Information System; Bladder Cancer Canada; Cancer Information Service (Japan); and GLOBOCAN (International Association of Cancer Registries). Websites were rereviewed during manuscript preparation to capture 2019 data where available. For situations where $\geq 1$ congress abstract reported the same study or data as a more recent full-text manuscript, all associated publications were captured and reported in the PRISMA diagram, but only data from the full-text manuscript were reported in the SLR to avoid duplication.

\section{INCLUSION CRITERIA}

Included studies were defined according to the PICOS (population, intervention, comparator, outcome, study type) framework using the following criteria: (a) population: adult patients aged $\geq 18$ years with UC or bladder cancer, further subdivided into those with bladder cancer, UC, or la/mUC; (b) intervention: any or none; (c) comparator: any or none; (d) outcomes: epidemiology (incidence and prevalence) or treatment patterns; and (e) study type: retrospective observational studies and retrospective or ad hoc analyses of data from clinical trials.

Included studies were from the following countries/ regions of interest: United States, Canada, Japan, United Kingdom, France, Germany, Italy, Spain, and Nordic countries.

\section{EXCLUSION CRITERIA}

Publications were excluded if they reported on animal, preclinical, pediatric, clinical trial, or economic modeling studies. Studies of primary urethral carcinoma, small cell carcinoma of the urinary bladder, upper tract UC, or studies in patients with any involvement of the urachus (if they did not also include data on patients with la/mUC) were also excluded to ensure data that related only to earlierstage disease such as nonmuscle invasive bladder cancer (NMIBC) and muscle invasive bladder cancer (MIBC) were not included. Reviews, meta-analyses, notes, case reports, comments, editorials, letters, or opinion articles were excluded, as were studies not published in English.

\section{LITERATURE SCREENING, DATA REPORTING, AND CRITICAL APPRAISAL}

Following initial searches, all citations were combined and duplications were removed. In level 1 screening, 2 independent reviewers assessed the titles and abstracts of identified publications against study inclusion/exclusion criteria. Full-text manuscripts retained after level 1 screening were then reviewed in level 2. Disagreements during level 1 or 
level 2 screening were resolved through discussion, with a third independent senior reviewer making the final decision if needed. All excluded studies were documented along with the reason for exclusion. Data collection from the included publications and epidemiology websites were reported in prespecified data extraction tables. Data were extracted by 1 reviewer and validated by a second reviewer for quality assurance. Critical appraisal of research quality was conducted using the Newcastle-Ottawa Scale to assess risk of bias. ${ }^{23}$

Among epidemiology publications, UC and la/mUC prevalence, incidence, and mortality or survival rates were reported. Among publications reporting treatments for patients with $\mathrm{UC}$ or la/mUC, the number and percentage of patients receiving cisplatin- versus carboplatin-containing regimens or classified as cisplatin-eligible versus -ineligible, patients treated with vinflunine, or patients treated with an IO agent (including the specific therapy where available), as well as those who received treatment after IO were reported. LOT for each of these treatments and number of patients discontinuing IO therapy were also reported.

\section{Results}

\section{SEARCH RESULTS}

Among 6,584 database references and 1,832 congress abstracts screened, 45 publications (29 manuscripts, 1 poster, 15 abstracts; reporting 37 unique studies) were retained after full-text review (Figure 1). The 29 manuscripts and 1 poster were critically appraised for risk of bias using the Newcastle-Ottawa Scale. Of these, 10 (33\%) had a low risk, 14 (47\%) moderate risk, and 6 (20\%) high risk; abstracts were not critically appraised. The sample size in these 30 studies ranged from 16 to 1,333 patients. The mean (SD) and median sample size were 174 (270) and 74.5, respectively; 7 studies had $<30$ patients, and 12 had $<50$ patients. Of these studies, 16 (53\%) were single center and thus may not have been representative of the population with mUC, $13(43 \%)$ did not control for potential confounders that could affect comparability of cohorts, 7 (23\%) included univariate between-group comparisons only, and 10 (33\%) used multivariate analyses. All 30 studies that were critically appraised were longitudinal and identified exposure (treatment) and outcome from medical charts or claims data.

Most publications meeting inclusion criteria $(n=45)$ reported data from the United States $(n=17)$, Japan $(n=8)$, and the United Kingdom ( $\mathrm{n}=5) ; 3$ reported data from Italy, 2 each from Germany and France, and 1 each from Canada, Spain, and Nordic countries; some reported on $>1$ country. Two studies were multinational, and 4 did not report the country, although they did report sufficient other data for inclusion. No epidemiological publications met inclusion criteria; although 8 publications were identified for data extraction, all were excluded due to outdated data $(n=4),{ }^{4,24-26}$ not reporting data for a country of interest $(n=2),{ }^{27,28}$ insufficient reporting $(n=1),{ }^{29}$ or other exclusion criteria $(n=1) \cdot{ }^{30}$ Because no published epidemiology data meeting study inclusion criteria were available, national and international epidemiology websites were used, as these provided the most recent data on incidence, prevalence, and mortality rates for bladder cancer. Data from these online resources were identified for bladder cancer only; no data were available for UC or la/mUC specifically.

All 45 included studies reported data on treatment patterns, which may have included specific treatment regimens or outcomes in patients who had received certain treatments by design. With respect to prespecified treatments of interest, 21 publications (20 unique studies) reported data on cisplatin versus non-cisplatin regimens, 14 (8 unique studies) on IO, and 9 (7 unique studies) on vinflunine. Other named agents identified in the studies that were not prespecified as of interest included gemcitabine monotherapy; gemcitabine plus carboplatin; gemcitabine plus paclitaxel; paclitaxel plus ifosfamide and nedaplatin; and paclitaxel plus carboplatin, docetaxel, and pemetrexed.

\section{INCIDENCE AND PREVALENCE}

Data extracted from national and international epidemiology websites are reported in Figures 2 and 3. Globally, the estimated annual bladder cancer incidence rate ranged from 6.9 per 100,000 in the United Kingdom to 15.9 per 100,000 in Italy.

\section{CISPLATIN- VERSUS NON-CISPLATIN-BASED REGIMENS}

A total of 31 papers reported on cisplatin versus non-cisplatin treatment regimens. Of these, 10 were not considered representative of treatment patterns because they had $<30$ patients $(n=5),{ }^{31-35}$ were clinical studies restricted to investigator-selected treatments $(\mathrm{n}=3),{ }^{36-38}$ or both $(\mathrm{n}=2){ }^{39,40}$ Twenty-one unique publications reported sufficient data to determine the percentage of patients receiving cisplatinversus non-cisplatin-based regimens (Table 1).

Approximately half of patients with la/mUC who received treatment received a first-line cisplatin-based regimen, as reported in 4 studies: $42 \%$ (United States: $n=1,703 ; 2004-$ 2011), ${ }^{41} 40 \%$ (United States: $\left.n=9,436 ; 2005-2015\right),{ }^{42} 50.9 \%$ (multinational: $\mathrm{n}=1,160 ; 2000-2013$ ), ${ }^{2}$ and $46 \%$ (multinational: $\mathrm{n}=1,020$; year not reported). ${ }^{43}$

A large variation in cisplatin use was observed both within and across countries. Among 10 studies from the United States, the proportion of patients receiving first-line 


\section{FIGURE 1 PRISMA Diagram of Study Selection}

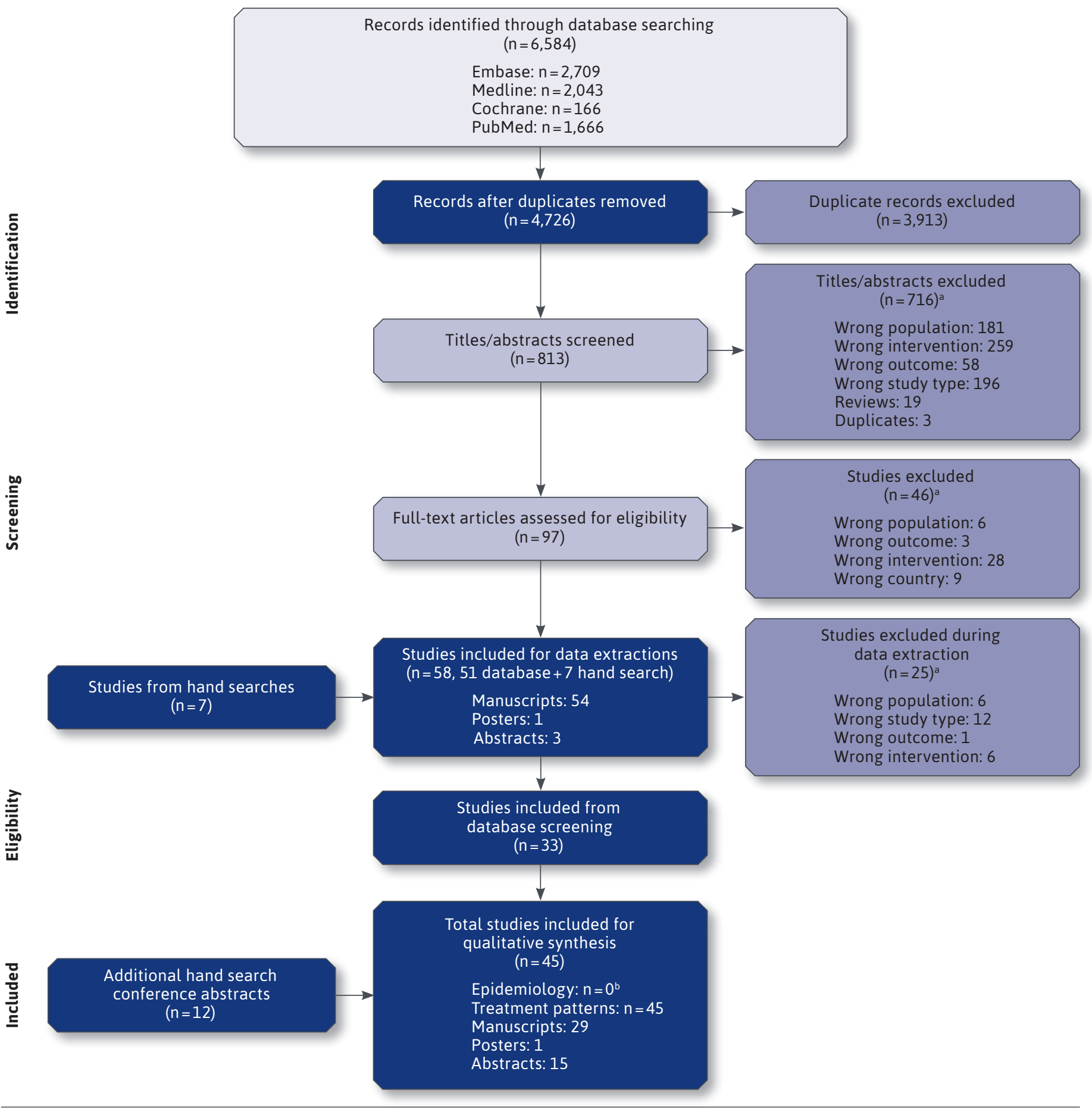

apapers not specific to la/mUC were excluded and coded as "wrong population"; papers outside the countries of interest were excluded and coded as "wrong country"; papers on surgery or radiation were excluded and coded "wrong intervention"; papers on pathology/histology/biomarkers/genotyping were excluded and coded "wrong outcome"; and clinical trials were excluded and coded as "wrong study type."

${ }^{b}$ No epidemiological publications reporting current data for qualitative synthesis were identified; supplemental searches of national and international epidemiology websites were conducted. 


\section{FIGURE 2 Estimated Annual Number of New Bladder Cancer Cases (2018) and 5-Year Prevalence (2018), by Country}

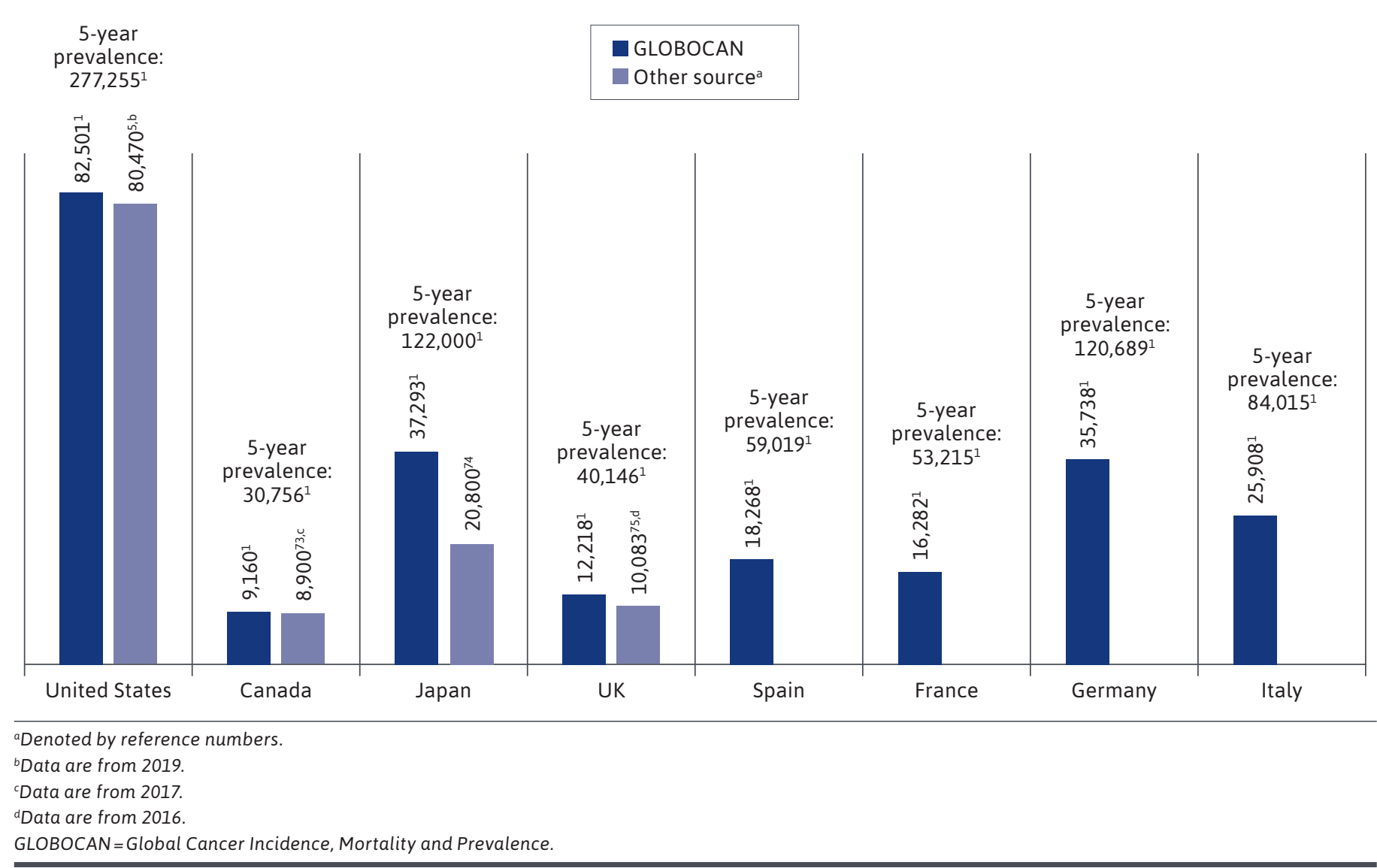

cisplatin-based treatment ranged from $18.4 \%$ to $60.2 \%{ }^{44,45}$ In the 2 qualifying studies from Japan, $>80.0 \%$ of patients received cisplatin-based treatment as first line. ${ }^{46,47}$ An additional study from Japan of 47 patients all receiving carboplatin-based chemotherapy reported that $62 \%$ of these patients were considered unfit to receive cisplatin; patients in this study had an average age of 77.1 years. ${ }^{38}$

In 3 studies, cisplatin eligibility was evaluated against established criteria. ${ }^{9}$ In these studies, a substantial proportion of cisplatin-eligible patients were not administered cisplatin. A multinational study of 1,160 patients found that $>25 \%$ of cisplatin-eligible patients did not receive cisplatin (patients received carboplatin or other therapies mainly due to advanced age and comorbidities), whereas nearly $35 \%$ of cisplatin-ineligible patients received cisplatinbased therapy. ${ }^{2}$ A study from the United States (2010-2014 data) reported that $48.7 \%$ of cisplatin-eligible patients were not treated with cisplatin (alternative therapies not provided), whereas $25.4 \%$ of cisplatin-ineligible patients received cisplatin anyway. ${ }^{48}$ In 1 retrospective Japanese study (not included in Table 1 due to the treatments being restricted by design), all 47 patients received carboplatinbased first-line treatment despite $38.3 \%$ of patients being cisplatin-eligible. ${ }^{38}$

\section{IMMUNO-ONCOLOGY AGENTS TARGETING PD-1/L1}

IO data were reported in 8 unique studies, 4 with real-world data, ${ }^{35,44,49,50} 3$ retrospective/post hoc analyses of patients who had received IO agents as part of a clinical trial, ${ }^{51-53}$ and 1 comparison of a clinical trial population with a realworld cohort. ${ }^{54}$ Seven of these studies provided detailed information (Table 2). Data were most commonly reported for IO therapies collectively, with treatment-specific data reported only for atezolizumab. ${ }^{50,54}$

The percentage of patients receiving IO therapy in real-world clinical practice was reported in 3 studies 


\section{FIGURE 3 Estimated Annual Incidence Rate (Number of Age-Standardized New Cases per 100,000), by Country (2018)}

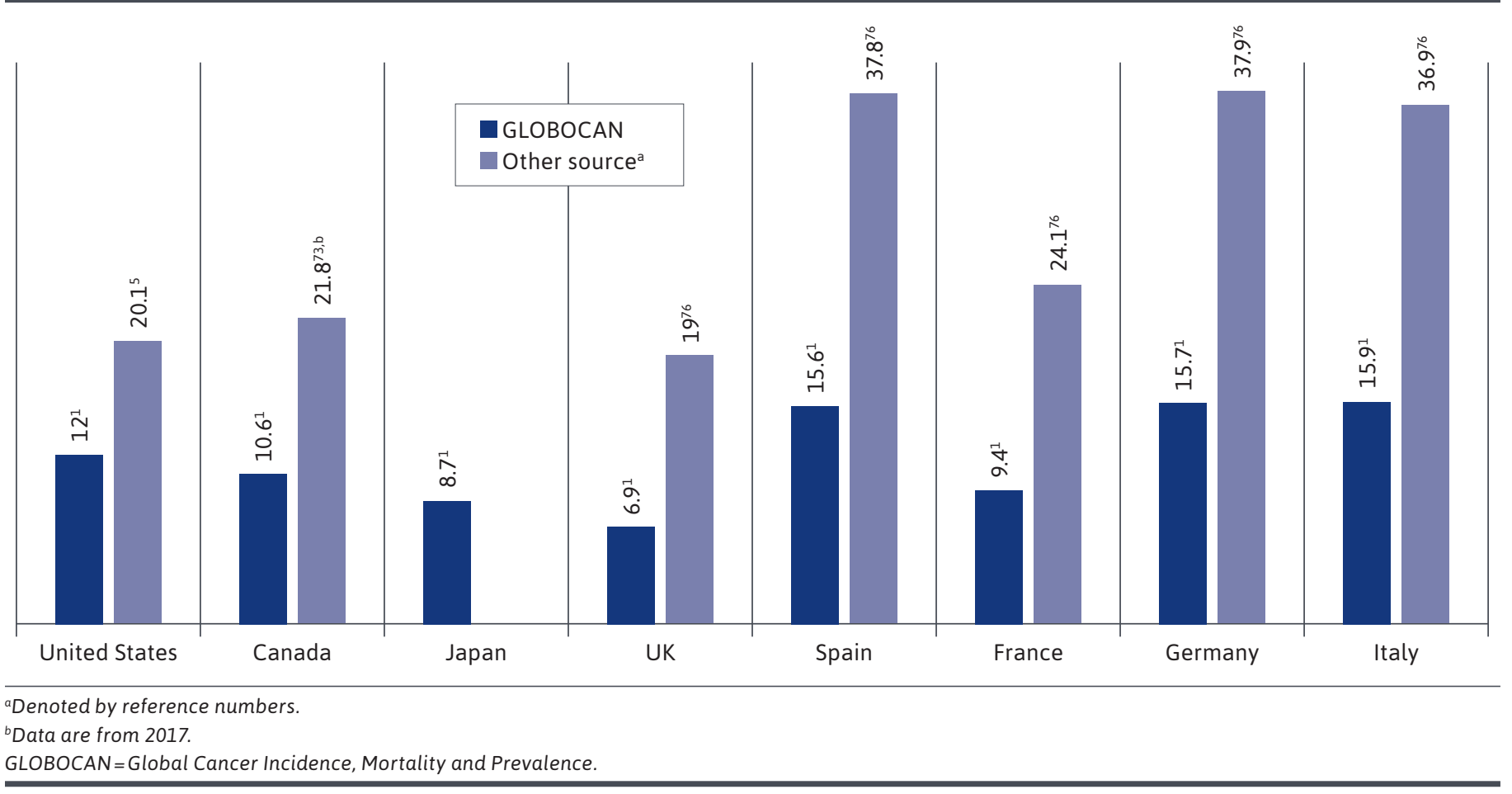

(1 additional study reported real-world data, but all included patients had received IO therapy ${ }^{50}$ ). Two of these studies, which included pre-IO approval data from Canada (20022016) and the United States (2011-2016), reported that only $1.3 \%$ and $4.4 \%$ of patients received first- and second-line IO, respectively. ${ }^{49,52}$ A more recent (2015-2017) study from the United States showed higher rates, with 7.9\%, 57.8\%, and $64.4 \%$ of patients receiving IO in first, second, and third lines, respectively. ${ }^{44}$

Real-world discontinuation rates were reported in 2 U.S. studies (discontinuation reasons not assessed). In the first study, IO discontinuation rates were $64.7 \%$ ( $n=22$ of 34 ), $54.0 \%$ ( $n=68$ of 126 ), and $41.4 \%$ ( $n=12$ of 29 ) for first, second, and third LOT, respectively. ${ }^{44}$ In this study, patients were reported to stay on IO therapy for a median of 3.0 months (95\% CI=2.3-5.9), 3.1 months (2.1-4.9), and 5.8 (1.6 to not reported) months by LOT, respectively. ${ }^{44}$ In the other study reporting discontinuation rates, patients receiving atezolizumab remained on therapy (LOT not reported) for a median of 2.7 months $(95 \% \mathrm{CI}=1.8-3.6)$, with $71 \%(\mathrm{n}=56$ of 79) discontinuing treatment..$^{50}$

The percentage of patients receiving subsequent therapy after discontinuing IO was reported in 2 studies and ranged from $25.0 \%$ to $35.5 \%{ }^{50,51}$ Post-IO treatments varied widely and included chemotherapy, IO, targeted therapies, clinical trial agents, or best supportive care. In 2 other studies, chemotherapy response rates were similar when administered before or after IO therapy in patients whose disease had relapsed. ${ }^{35,53}$ In a study of 115 patients receiving IO sequenced with carboplatin chemotherapy, the rates of partial response (PR)/complete response (CR) to carboplatin-based chemotherapy were $49 \%$ PR ( $0 \%$ CR) when administered before IO, compared with $40 \%$ PR (4\% CR) when administered after IO. ${ }^{53}$ In a second study, patients receiving chemotherapy after IO had a PR of $64 \%(n=9$ of $14)$ if they were chemotherapy-naive and a PR of $21 \%(n=3$ of 14) if they had previously discontinued a chemotherapy treatment. ${ }^{35}$

\section{VINFLUNINE STUDIES}

Vinflunine data were presented in 7 unique studies (Supplementary Table 1, available in online article). ${ }^{31,33,34,55-58}$ All 7 studies were retrospective analyses reporting data from real-world clinical practice in Europe.

Real-world treatment patterns by LOT were reported in 1 study (2009-2016) from Germany $(\mathrm{N}=435)$, of which $3 \%$, 


\section{TABLE 1 Studies Reporting First-Line Cisplatin- Versus Non-Cisplatin-Based Regimens, by Country} (Studies with $\geq 30$ Patients and $<10 \%$ Missing Data)

\begin{tabular}{|c|c|c|c|c|c|c|}
\hline $\begin{array}{l}\text { Author, } \\
\text { Year }\end{array}$ & $\begin{array}{l}\text { Country and } \\
\text { Data Source }\end{array}$ & $\begin{array}{l}\text { Publication } \\
\text { Type }\end{array}$ & Study Period & $\begin{array}{l}\text { Patients with } \\
\text { Drug Data, } \mathbf{n}\end{array}$ & LOT & $\begin{array}{l}\text { Patients with Cisplatin- vs. } \\
\text { Non-Cisplatin-Based Regimen, } \mathrm{n}(\%)\end{array}$ \\
\hline $\begin{array}{l}\text { Bamias A, } \\
2018^{2}\end{array}$ & $\begin{array}{l}\text { Multinational RISC } \\
29 \text { centers }\end{array}$ & Manuscript & $2000-2013$ & $\begin{array}{c}\text { TCCa: } \\
1,160 \\
\text { (of } 1,174 \text { ) with } \\
\text { drug data } \\
\text { TCC + mixed: } \\
1,260 \\
\text { (of } 1,278 \text { ) with } \\
\text { drug data }\end{array}$ & $1 \mathrm{~L}$ & $\begin{array}{l}\text { TCC } \\
\text { Cis: } 591(50.3) \\
\text { Non-cis: } 569(48.5) \\
\text { Car: } 348(29.6) \\
\text { Other: } 221(18.8) \\
\text { NR: } 14(1.2) \\
\text { TCC + mixed } \\
\text { Cis: } 636(50.5) \\
\text { Non-cis: } 624(49.5) \\
\text { Car: } 377(29.6) \\
\text { Other: } 247(19.6) \\
\text { NR: } 18 \text { (1.4) }\end{array}$ \\
\hline $\begin{array}{l}\text { Necchi A, } \\
2018^{43}\end{array}$ & $\begin{array}{l}\text { Multinational } \\
\text { (RISC) } \\
\text { Number of centers } \\
\text { not reported }\end{array}$ & Abstract & NR & 472 & $1 \mathrm{~L}$ & $\begin{array}{l}\text { Cis: } 338 \text { (71.6) } \\
\text { Non-cis: Car: } 134 \text { (28.4) }\end{array}$ \\
\hline $\begin{array}{l}\text { Locke JA, } \\
2016^{66}\end{array}$ & $\begin{array}{l}\text { Multinational } \\
12 \text { centers }\end{array}$ & Manuscript & $1995-2014$ & 145 & $1 \mathrm{~L}$ & $\begin{array}{l}\text { Cis: } 91 \text { (62.8) } \\
\text { Non-cis: } 54 \text { (37.2) }\end{array}$ \\
\hline $\begin{array}{l}\text { Galsky MD, } \\
2018^{41}\end{array}$ & $\begin{array}{l}\text { United States } \\
\text { SEER-Medicare } \\
\text { database }\end{array}$ & Manuscript & 2004-2011 & 717 & $1 \mathrm{~L}$ & $\begin{array}{l}\text { Cis: } 192(26.8) \\
\text { Non-cis: } 465(64.8) \\
\text { NR: } 60(8.4)\end{array}$ \\
\hline $\begin{array}{l}\text { Barata PC, } \\
2018^{50}\end{array}$ & $\begin{array}{l}\text { United States } \\
\text { Single center } \\
\text { (Cleveland Clinic) }\end{array}$ & Manuscript & 2016-2017 & 79 & $1 \mathrm{~L}, 2 \mathrm{~L}, 3 \mathrm{~L}$ & $\begin{array}{l}\text { Cis: } 34 \text { (43.0) } \\
\text { Non-cis: } 45 \text { (57.0) } \\
\text { Atezolizumab: } 20 \text { (25.3) } \\
\text { Clinical trial IO: } 2 \text { (2.5) } \\
\text { Car/other: } 23 \text { (29.1) } \\
\text { (cannot differentiate LOT) }\end{array}$ \\
\hline $\begin{array}{l}\text { Doshi G, } \\
2018^{44}\end{array}$ & $\begin{array}{l}\text { United States } \\
\text { U.S. Oncology } \\
\text { Network, } \\
\text { iKnowMed }\end{array}$ & Poster & $2015-2017$ & 430 & $1 \mathrm{~L}$ & $\begin{array}{l}\text { Cis: } 79(18.4) \\
\text { Non-cis: } 351(81.6) \\
\text { Car: } 154(35.8) \\
\text { IO: } 34(7.9) \\
\text { Other: } 163(37.9)\end{array}$ \\
\hline $\begin{array}{l}\text { Malangone- } \\
\text { Monaco E, } \\
2017^{42}\end{array}$ & $\begin{array}{l}\text { United States } \\
\text { U.S. insurance } \\
\text { claims database }\end{array}$ & Abstract & 2005-2015 & 3,750 & $1 \mathrm{~L}$ & $\begin{array}{l}\text { Cis: } 935 \text { (24.9) } \\
\text { Non-cis: } 2,815(75.1) \\
\text { Car: } 1,505(40.1) \\
\text { Other: } 1,310(34.9)\end{array}$ \\
\hline $\begin{array}{l}\text { Kunthur A, } \\
2017^{45}\end{array}$ & $\begin{array}{l}\text { United States } \\
\text { VA central cancer } \\
\text { registry }\end{array}$ & Abstract & $2000-2010$ & 196 & $1 \mathrm{~L}$ & $\begin{array}{l}\text { Cis: } 118 \text { (60.2) (100\% gem-cis) } \\
\text { Non-cis: } 78 \text { (39.8) (100\% gem-car) }\end{array}$ \\
\hline
\end{tabular}




\section{TABLE 1 Studies Reporting First-Line Cisplatin- Versus Non-Cisplatin-Based Regimens, by Country} (Studies with $\geq 30$ Patients and $<10 \%$ Missing Data) (continued)

\begin{tabular}{|c|c|c|c|c|c|c|}
\hline $\begin{array}{l}\text { Author, } \\
\text { Year }\end{array}$ & $\begin{array}{l}\text { Country and } \\
\text { Data Source }\end{array}$ & $\begin{array}{c}\text { Publication } \\
\text { Type }\end{array}$ & Study Period & $\begin{array}{l}\text { Patients with } \\
\text { Drug Data, } \mathbf{n}\end{array}$ & LOT & $\begin{array}{l}\text { Patients with Cisplatin- vs. } \\
\text { Non-Cisplatin-Based Regimen, } \mathbf{n}(\%)\end{array}$ \\
\hline $\begin{array}{l}\text { Flannery K, } \\
2017^{67}\end{array}$ & $\begin{array}{l}\text { United States } \\
\text { Electronic } \\
\text { health records } \\
\text { of McKesson } \\
\text { Specialty Health/ } \\
\text { U.S. Oncology } \\
\text { Network }\end{array}$ & Abstract & $2010-2014$ & 1,155 & $1 \mathrm{~L}$ & $\begin{array}{l}\text { Gem-cis: } 310 \text { (26.8) } \\
\text { Car: } 409 \text { (35.4) } \\
\text { Gem: } 96 \text { (8.3) } \\
\text { NR (may include cis): } 341 \text { (29.5) }\end{array}$ \\
\hline $\begin{array}{l}\text { Fisher M, } \\
201768, \mathrm{~b}\end{array}$ & $\begin{array}{l}\text { United States } \\
10 \text { U.S. community } \\
\text { oncology } \\
\text { practices }\end{array}$ & Abstract & $2008-2015$ & $\begin{array}{c}508 \mathrm{mBC} \\
286(56.3 \%) \text { had } \\
1 \mathrm{~L} \text { platinum }\end{array}$ & $1 \mathrm{~L}$ & $\begin{array}{l}\text { Cis: Gem-cis: NR (17.0) } \\
\text { Non-cis: Gem-car: NR (23.6) } \\
\text { Unknown: NR (59.4) }\end{array}$ \\
\hline $\begin{array}{l}\text { Zargar- } \\
\text { Shoshtari K, } \\
2016^{69, c}\end{array}$ & $\begin{array}{l}\text { United States } \\
\text { Single institution } \\
\text { database }\end{array}$ & Manuscript & $2001-2013$ & $\begin{array}{c}88 \\
\text { (neoadjuvant } \\
\text { data) } \\
29 \text { with } \\
\text { adjuvant data }\end{array}$ & Adjuvant & $\begin{array}{l}\text { Cis: } 8 \text { (27.6) } \\
\text { Non-cis: } 21(72.4) \\
\text { Car: } 16(55.2) \\
\text { Other: } 5(17.2)\end{array}$ \\
\hline $\begin{array}{l}\text { Bambury RM, } \\
2015^{70}\end{array}$ & $\begin{array}{l}\text { United States } \\
\text { Institutional } \\
\text { database } \\
\text { (Memorial Sloan } \\
\text { Kettering Cancer } \\
\text { Center) }\end{array}$ & Manuscript & $2008-2013$ & 129 & $1 \mathrm{~L}, 2 \mathrm{~L}$ & $\begin{array}{l}\text { Cis: } 74 \text { (57.4) } \\
\text { Non-cis: Car: } 55 \text { (42.6) } \\
\text { (cannot differentiate LOT) }\end{array}$ \\
\hline $\begin{array}{l}\text { Yelfimov DA, } \\
2014^{71}\end{array}$ & $\begin{array}{l}\text { United States } \\
\text { Single center } \\
\text { (Mayo Clinic } \\
\text { Minnesota) }\end{array}$ & Manuscript & $1980-2005$ & 80 & Adjuvant & $\begin{array}{l}\text { Cis: } 43 \text { (53.8) } \\
\text { Non-cis: Car: } 17(21.2) \\
\text { Unknown: } 20 \text { (25.0) }\end{array}$ \\
\hline $\begin{array}{l}\text { Kikuchi E, } \\
2016^{46}\end{array}$ & $\begin{array}{l}\text { Japan } \\
17 \text { centers }\end{array}$ & Manuscript & 2004-2010 & 107 with mUC & $1 \mathrm{~L}$ & $\begin{array}{l}\text { Cis: } 87(81.3) \\
\text { Non-cis: } 20(18.7) \\
\text { Car: } 10(9.4) \\
\text { Other: } 10(9.4)\end{array}$ \\
\hline $\begin{array}{l}\text { Muto S, } \\
2015^{47, d}\end{array}$ & $\begin{array}{l}\text { Japan } \\
\text { Teikyo University } \\
\text { Hospital, Tokyo } \\
\text { and Dokkyo } \\
\text { Medical University } \\
\text { Hospital, Tochigi }\end{array}$ & Manuscript & $2008-2014$ & \begin{tabular}{|c}
$\mathrm{N}=66:$ \\
33 (2L gem \\
monotherapy) \\
33 (2L best \\
supportive care \\
control group)
\end{tabular} & $1 \mathrm{~L}$ & $\begin{array}{l}\text { Cis: } 58 \text { (87.9) } \\
\text { Gem group: } 28 \text { (84.8) } \\
\text { Control: } 30 \text { (90.9) } \\
\text { Non-cis: } 8 \text { (12.1) } \\
\text { Gem group: } 5 \text { (15.2) } \\
\text { Control: } 3 \text { (9.1) }\end{array}$ \\
\hline $\begin{array}{l}\text { Yip SM, } \\
2018^{52}\end{array}$ & $\begin{array}{l}\text { Canada } \\
5 \text { cancer centers } \\
\text { in Alberta, Canada }\end{array}$ & Manuscript & $2002-2016$ & 233 & $1 \mathrm{~L}$ & $\begin{array}{l}\text { Cis: } 130(55.8) \\
\text { Non-cis: } 103(44.2) \\
\text { Car: } 87(37.3) \\
\text { Other: } 16(6.9)\end{array}$ \\
\hline $\begin{array}{l}\text { Niegisch G, } \\
2018^{55}\end{array}$ & $\begin{array}{l}\text { Germany } \\
\text { GermanOncology } \\
\text { (GO) network, } \\
\text { office-based sites, } \\
\text { nonacademic } \\
\text { clinics, and } \\
\text { academic clinics }\end{array}$ & Manuscript & $2009-2016$ & 435 & $1 \mathrm{~L}$ & $\begin{array}{l}\text { Cis: } 309(71.0) \\
\text { Non-cis: } 126(29.0) \\
\text { Car: } 56(12.9) \\
\text { Other: } 70(16.1)\end{array}$ \\
\hline
\end{tabular}




\section{TABLE 1 Studies Reporting First-Line Cisplatin- Versus Non-Cisplatin-Based Regimens, by Country} (Studies with $\geq 30$ Patients and $<10 \%$ Missing Data) (continued)

\begin{tabular}{|c|c|c|c|c|c|c|}
\hline $\begin{array}{l}\text { Author, } \\
\text { Year }\end{array}$ & $\begin{array}{l}\text { Country and } \\
\text { Data Source }\end{array}$ & $\begin{array}{c}\text { Publication } \\
\text { Type }\end{array}$ & Study Period & $\begin{array}{l}\text { Patients with } \\
\text { Drug Data, } \mathbf{n}\end{array}$ & LOT & $\begin{array}{l}\text { Patients with Cisplatin- vs. } \\
\text { Non-Cisplatin-Based Regimen, n (\%) }\end{array}$ \\
\hline $\begin{array}{l}\text { Passalacqua R, } \\
2017^{56}\end{array}$ & $\begin{array}{l}\text { Italy } \\
28 \text { Italian cancer } \\
\text { centers }\end{array}$ & Manuscript & 2011-2014 & 217 & $1 \mathrm{~L}$ & $\begin{array}{l}\text { Cis: } 122(56.2) \\
\text { Non-cis: } 95(43.8) \\
\text { Car: } 91(42.0) \\
\text { Other: } 4(1.8)\end{array}$ \\
\hline $\begin{array}{l}\text { Laurent M, } \\
2016^{72}\end{array}$ & $\begin{array}{l}\text { France } \\
\text { AGEVIM } \\
\text { multicenter } \\
\text { cohort }\end{array}$ & Manuscript & 1999-2011 & $\begin{array}{c}193 \text { with } \mathrm{mBC} \\
178(92.2 \%) \\
\text { with } \mathrm{mUC}\end{array}$ & $1 \mathrm{~L}$ & $\begin{array}{l}\text { Among } 193 \text { mBC patients } \\
\text { Cis: } 84 \text { (43.5) } \\
\text { Non-cis: } 109 \text { (56.5) } \\
\text { Car/oxaliplatin: } 70(36.3) \\
\text { Other: } 39 \text { (20.2) }\end{array}$ \\
\hline $\begin{array}{l}\text { Hussain S, } \\
2017^{58}\end{array}$ & $\begin{array}{l}\text { United Kingdom } \\
\text { and Ireland } \\
9 \text { sites }\end{array}$ & Manuscript & 1999-2013 & 43 & $1 \mathrm{~L}$ & $\begin{array}{l}\text { Cis: } 13 \text { (30.2) } \\
\text { Non-cis: Car: } 16 \text { (37.2) }\end{array}$ \\
\hline $\begin{array}{l}\text { Holmsten K, } \\
2016^{57}\end{array}$ & $\begin{array}{l}\text { Denmark and } \\
\text { Sweden }\end{array}$ & Manuscript & 2010-2013 & 100 & $1 \mathrm{~L}$ & $\begin{array}{l}\text { Cis: } 76 \text { (76.0) } \\
\text { Non-cis: } 24(24.0) \\
\text { Car: } 17(17.0) \\
\text { Other: } 7(7.0)\end{array}$ \\
\hline
\end{tabular}

aUC is also known as TCC.

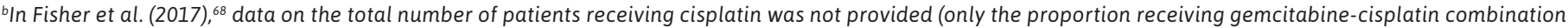
was reported).

'Zargar-Shoshtari et al. (2016) ${ }^{69}$ reported on 29 patients receiving cisplatin versus non-cisplatin adjuvant therapy (2001-2013).

dIn Muto et al. (2015), ${ }^{47}$ patients received either $2 \mathrm{~L}$ gemcitabine maintenance therapy or best supportive care.

$1 \mathrm{~L}=$ first line; $2 \mathrm{~L}=$ second line; $3 \mathrm{~L}=$ third line; $\mathrm{AGEVIM}=\mathrm{AGE}$-Vessle-chiMiotherapie or Age-Bladder-Chemotherapy; $c$ ar =carboplatin; $c$ is =cisplatin; gem = gemcitabine; $1 O=$ immuno-oncology; $L O T=$ line of therapy; $m B C=$ metastatic bladder cancer; $m U C=$ metastatic urothelial carcinoma; $N R=$ not reported;

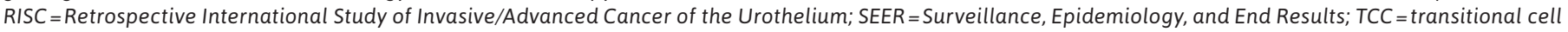
carcinoma; $\cup C=$ urothelial carcinoma; $V A=$ Veterans Administration.

$47.2 \%$, and $32.4 \%$ of patients were treated with vinflunine in the first, second, and third line, respectively. ${ }^{55}$ In the remaining 6 studies, all patients received vinflunine; sample sizes ranged from $19^{33}$ to $217 .{ }^{56}$ In a study from the United Kingdom/Ireland, vinflunine was provided as second-line treatment to 49 patients in a Free of Charge Program, as it was not available through the National Health Service. ${ }^{58}$

\section{PATIENT ESTIMATES}

The findings from this SLR were summarized into a flow model to estimate the number of U.S. patients with la/mUC whose disease progressed after receipt of IO therapy, including those who received subsequent therapy. Approximately 22,542 patients are diagnosed with or progress to la/mUC annually, of whom 11,600 received first-line therapy. An estimated 1,795 patients would be eligible to receive therapy post-IO, including 1,352 patients who progress after chemotherapy and an IO agent (Supplementary Figure 1, available in online article). Due to the extent of missing data for numerous inputs, an accurate estimate of the number of patients receiving IO for other countries of interest was not possible.

\section{Discussion}

With the emergence of novel therapy options for patients with la/mUC, assessment of the current epidemiology and treatment landscape is important to provide an evidence base for their evaluation. This SLR was conducted to identify and summarize the published literature on UC and la/mUC incidence and prevalence rates and la/mUC treatment patterns and to identify evidence gaps. A total of 45 publications on la/mUC treatment patterns, comprising 37 unique studies, were retained after full-text review; no studies on the epidemiology of UC and la/mUC were found and epidemiological data were obtained from the national and international websites. The limited number of retained 


\begin{tabular}{|c|c|c|c|c|c|c|c|}
\hline $\begin{array}{l}\text { Author, } \\
\text { Year }\end{array}$ & Agent & $\begin{array}{l}\text { Country and } \\
\text { Data Source }\end{array}$ & Study Period & $\begin{array}{l}\text { Patients with } \\
\text { Drug Data, } \mathbf{n}\end{array}$ & $\begin{array}{c}\text { Patients with I0, } \\
\text { by LOT, } \mathrm{n}(\%)\end{array}$ & $\begin{array}{l}\text { Patients with } \\
\text { Progression, } \\
\text { Discontinuation, } \\
\text { or Response, } \\
\text { by IO LOT }\end{array}$ & $\begin{array}{l}\text { Patients with } \\
\text { Subsequent } \\
\text { Treatment Among } \\
\text { Those Who } \\
\text { Discontinue IO, } \\
\text { by IO LOT, n (\%) }\end{array}$ \\
\hline $\begin{array}{l}\text { Doshi G, } \\
2018^{44}\end{array}$ & $10^{a}$ & $\begin{array}{l}\text { United States } \\
\text { U.S. Oncology } \\
\text { Network, iKnowMed }\end{array}$ & 2015-2017 & $\begin{array}{l}\text { 1L: } 430 \\
\text { 2L: } 218 \\
3 L: 45\end{array}$ & $\begin{array}{l}\text { 1L: } 34(7.9) \\
\text { 2L: } 126(57.8) \\
\text { 3L: } 29(64.4)\end{array}$ & $\begin{array}{l}\text { Tx failure event } \\
\text { 1L: } 22(64.7 \%) ; \\
\text { median TTF } \\
\text { 13.0 weeks } \\
\text { 2L: } 68(53.9 \%) ; \\
\text { median TTF } \\
\text { 13.3 weeks } \\
\text { 3L: } 12(41.4 \%) ; \\
\text { median TTF } \\
\text { 25.3 weeks }\end{array}$ & $N R$ \\
\hline $\begin{array}{l}\text { Vander } \\
\text { Velde N, } \\
2019^{54}\end{array}$ & Atezolizumab & $\begin{array}{l}\text { United States } \\
\text { IMvigor210 and VHA }\end{array}$ & 2006-2017 & $\begin{array}{l}\text { 1L: } 392 \\
110 \text { in } \\
\text { IMvigor210 }\end{array}$ & $\begin{array}{l}\text { 1L: } 110(100.0) \\
\text { atezolizumabc }^{c}\end{array}$ & $\begin{array}{l}\text { NR } \\
\text { Mean duration } \\
\text { of treatment: } \\
11.0 \text { months }\end{array}$ & NR \\
\hline $\begin{array}{l}\text { Barata PC, } \\
2018^{50}\end{array}$ & Atezolizumab & $\begin{array}{l}\text { United States } \\
\text { Single center } \\
\text { (Cleveland Clinic) }\end{array}$ & NR & $\begin{array}{l}\text { 1L: } 20 \\
2 \mathrm{~L}: 47 \\
3 \mathrm{~L}: 9 \\
4 \mathrm{~L}: 3\end{array}$ & $\begin{array}{l}\text { 100\% received } \\
\text { atezolizumabc } \\
\text { 1L: } 20(25.3) \\
\text { 2L: } 47(59.5) \\
\text { 3L: } 9(11.4) \\
\text { 4L: } 3(3.8)\end{array}$ & $\begin{array}{l}\text { NR } \\
\text { Median duration } \\
\text { of treatment all } \\
\text { LOT: } 2.7 \text { months, } \\
56(70.9 \%) \\
\text { discontinued }\end{array}$ & $\begin{array}{l}25 \% \text { of } 56 \\
\text { discontinued } \\
\text { patients (LOT not } \\
\text { specified) }\end{array}$ \\
\hline $\begin{array}{l}\text { Sonpavde G, } \\
2017^{51}\end{array}$ & $\begin{array}{l}\text { Anti-PD-1/L1 } \\
\text { therapy }^{\mathrm{a}}\end{array}$ & $\begin{array}{l}\text { United States, Italy, } \\
\text { and United Kingdom } \\
4 \text { institutions } \\
\text { (Dana-Farber } \\
\text { Cancer Institute, } \\
\text { Comprehensive } \\
\text { Cancer Centers of } \\
\text { Nevada, Istituto } \\
\text { Nazionale dei } \\
\text { Tumori, and Barts } \\
\text { Cancer Institute) }\end{array}$ & NR & All LOT: 62 & NR & $\begin{array}{l}\text { By design: } 100 \% \\
\text { progressed in } \\
\text { all LOT } \\
\text { During IO } \\
\text { therapy, } \\
\text { progressive } \\
\text { disease: } \\
31(50.0 \%) \\
\text { Post-1O therapy, } \\
\text { progressive } \\
\text { disease: } 5(22.7 \%)\end{array}$ & $\begin{array}{l}22(35.5) \text { of } \\
62 \text { who } \\
\text { discontinued } \\
\text { (any LOT) } \\
\text { Study does not } \\
\text { report patients } \\
\text { who responded } \\
\text { to PD-1/PD-L1 } \\
\text { inhibitor }\end{array}$ \\
\hline
\end{tabular}

continued on next page

publications likely reflects the relatively small volume of patients with la/mUC, as well as the lack of treatment options for these patients (besides chemotherapies) until 2016.

Bladder cancer was the tenth most common cancer, with an estimated 549,393 new cases worldwide in 2019. ${ }^{1}$ In the United States, there are an estimated $80,400^{5}$ to $82,501^{1}$ new bladder cancer cases annually, with an estimated 5-year prevalence of $277,255 .{ }^{1}$ Among the countries investigated, the highest bladder cancer incidence rates (per 100,000) were seen in Italy (15.9-36.9), Germany (15.7-37.9), and Spain (15.6-37.8) ${ }^{1,59}$; the United Kingdom had the lowest incidence rates (6.9-19.0 per 100,000)., ${ }^{1,59}$ Although epidemiology websites provided clear estimates of bladder cancer incidence and prevalence, little data were available pertaining to la/mUC. Although studies published before the SLR study period report rates of progression from early-stage disease to la/mUC, ${ }^{60,61}$ no new studies were found in this SLR suggesting there is a lack of up-to-date information. Additional studies in large databases or patient registries are needed to identify the country-specific epidemiology of $\mathrm{UC}$ and $\mathrm{la} / \mathrm{mUC}$, including the rates of progression from early-stage disease. This evidence gap is especially important to address given the potential effect of new and emerging therapies on disease progression. 


\begin{tabular}{|c|c|c|c|c|c|c|c|}
\hline $\begin{array}{l}\text { Author, } \\
\text { Year }\end{array}$ & Agent & $\begin{array}{l}\text { Country and } \\
\text { Data Source }\end{array}$ & Study Period & $\begin{array}{l}\text { Patients with } \\
\text { Drug Data, n }\end{array}$ & $\begin{array}{l}\text { Patients with IO, } \\
\text { by LOT, } \mathbf{n}(\%)\end{array}$ & $\begin{array}{l}\text { Patients with } \\
\text { Progression, } \\
\text { Discontinuation, } \\
\text { or Response, } \\
\text { by IO LOT }\end{array}$ & $\begin{array}{c}\text { Patients with } \\
\text { Subsequent } \\
\text { Treatment Among } \\
\text { Those Who } \\
\text { Discontinue IO, } \\
\text { by IO LOT, n (\%) }\end{array}$ \\
\hline $\begin{array}{l}\text { Szabados B, } \\
2018^{35}\end{array}$ & $\begin{array}{c}\text { Anti-PD-1/L1 } \\
\text { therapy }^{\mathrm{a}}\end{array}$ & $\begin{array}{l}\text { United Kingdom and } \\
\text { Netherlands } \\
2 \text { institutional } \\
\text { databases from } \\
\text { clinical trials } \\
\text { (Barts Health and } \\
\text { Netherlands Cancer } \\
\text { Institute) }\end{array}$ & NR & All LOT: 28 & $\begin{array}{l}\text { By design: } \\
\text { 1L: } 14(50.0) \\
\text { 2L: } 14(50.0) \\
\text { IO/CT } \\
\text { sequencing } \\
\text { study }\end{array}$ & $\begin{array}{l}\text { By design: } \\
28(100.0 \%) \\
\text { progressed in } \\
\text { all LOT } \\
\text { Median } \\
\text { follow-up: } \\
8.2 \text { (IQR, } \\
6.5-11.3 \text { ) months }\end{array}$ & $\begin{array}{l}\text { By design, } 100 \% \\
\text { of pts received } \\
\text { subsequent CT }\end{array}$ \\
\hline $\begin{array}{l}\text { Simeone JC, } \\
2018^{49}\end{array}$ & $10^{\mathrm{a}}$ & $\begin{array}{l}\text { United States } \\
\text { Flatiron Oncology }\end{array}$ & $2011-2016$ & $\begin{array}{l}476 \\
\text { 1L: } 476 \\
2 L: 476\end{array}$ & $\begin{array}{l}\text { 1L: } 0 \text { (all } \\
\text { platinum- } \\
\text { based) } \\
\text { 2L: } 21(4.4)\end{array}$ & NR & NR \\
\hline $\begin{array}{l}\text { Wei X, } \\
2018^{53}\end{array}$ & $\begin{array}{c}\text { Anti-PD-1/L1 } \\
\text { therapy }^{\mathrm{a}}\end{array}$ & $\begin{array}{l}\text { NR; multinational/ } \\
\text { multiple institutions } \\
\text { assumed based on } \\
\text { author list }\end{array}$ & NR & All LOT: 115 & $\begin{array}{l}\text { 1L: } 33(28.7) \\
\text { 2L: } 82(71.3) \\
\text { IO/car regimen } \\
\text { sequencing } \\
\text { study }\end{array}$ & $\begin{array}{l}\text { 1L: PR: } 9 \% \text {; } \\
\text { CR: } 0 \% \\
\text { 2L: PR: } 22 \% \text {; } \\
\text { CR: } 4 \%\end{array}$ & $\begin{array}{l}\text { By design, } \\
100 \% \text { of pts with } \\
\text { 1L IO received } \\
\text { 2L car-based } \\
\text { treatment }\end{array}$ \\
\hline
\end{tabular}

Note: Yip et al. (2018), 52 a Canadian study not listed here, reported that in 2L, 1 of 79 patients received IO.

${ }^{a}$ Agent not specified.

bStudy compared cisplatin-ineligible patients with mUC from the phase 2 trial IMvigor210 (treated with atezolizumab) with patients from the VHA health care system (treated according to normal clinical practice) using IMvigor210 eligibility criteria applied by proxy measurements.

'Non-IO data not included.

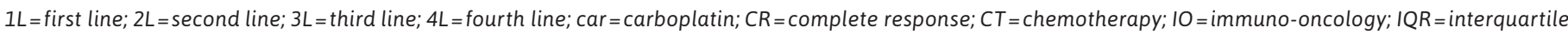

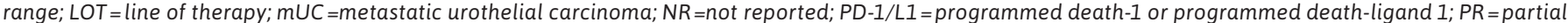
response; $p t s=$ patients; $T T F=$ time to treatment failure; $T x=$ treatment; VHA=Veterans Health Administration.

A large variation in cisplatin use was observed both within and among countries, with a trend toward lower cisplatin use in studies with the most recent data periods (evaluating data post-2010, cisplatin use was $43.0 \%$ for 2016-2017 [United States], ${ }^{50}$ 56.2\% for 2011-2014 [Italy], ${ }^{56}$ and $76.0 \%$ for 2010-2013 [Denmark and Sweden] ${ }^{57}$ ), although studies spanned multiple years across different countries making conclusions difficult to establish. This trend was further substantiated by a recent study identified outside the SLR period that showed a decrease from $53 \%$ to $33 \%$ in the proportion of eligible patients who received first-line cisplatin-based therapy in the United States between 2015 and 2018. ${ }^{62}$ The data also suggest that patients are not being consistently treated according to recommendations based on cisplatin-eligibility criteria. For example, a large multinational analysis of Retrospective International Study of Invasive/Advanced Cancer of the Urothelium (RISC) data established that eligibility criteria accounted for only $56 \%$ of the cases in which the investigator provided a reason for not using cisplatin, ${ }^{2}$ suggesting other considerations in addition to cisplatin-eligibility are important to treatment decisions in real-world clinical practice. It should be noted that patients included in this analysis were treated before the publication of cisplatin-eligibility criteria by Galsky et al. (2011). ${ }^{9}$ Much of the identified published evidence on cisplatin versus non-cisplatin use was outdated and therefore may not reflect current treatment patterns. Further, not all data were specific to patients with la/mUC, and studies may have reported on the overall population with UC or only some stages/extent of disease. The most recent data (2015-2017) available were from the United States, ${ }^{44}$ with limited data overall on cisplatin versus non-cisplatin use outside the United States and Japan.

Given IO therapy for la/mUC has only been approved since 2016, few studies reporting real-world treatment patterns have been published to date. Overall, the use of IO therapy has been increasing, as reflected by higher IO treatment rates in more recent studies. This trend 
was confirmed in an additional study published after the SLR study period that found the quarterly proportion of patients who received first-line IO increased from $1.4 \%$ to $42.0 \%$ between 2015 and 2018. ${ }^{62}$ In a separate analysis by Parikh et al. (2019), IO use decreased from $51.9 \%$ to $30.3 \%$ between May 2018 and January 2019 following the FDA's decision to restrict the first-line use of atezolizumab and pembrolizumab to cisplatin-ineligible patients with PD-L1-positive tumors. ${ }^{63}$ Nonetheless, the overall increase in the use of IO for patients with la/mUC represents a major shift in clinical practice that is continuing to evolve.

Despiteincreased use, most patients discontinue IO, with 1 study reporting that up to $71 \%$ of patients discontinued atezolizumab across all LOT. ${ }^{50}$ Median IO treatment duration reported in clinical trials is 2-3 months, ${ }^{15-17}$ whereas studies identified in the SLR indicated patients often discontinued IO treatment within 3-6 months in the real-world setting. ${ }^{44,50}$ Limited data have been published on IO treatment duration in the real-world setting since the SLR was conducted. One recent analysis reported a median duration of $\mathrm{IO}$ treatment ranging from 2.1 to 2.8 months. ${ }^{64}$ Few patients receive subsequent therapy following IO, reflecting a persisting unmet need, in particular for those patients whose disease progressed after IO treatment. ${ }^{50,51}$ The emergence of novel therapies may help fill this unmet need. For example, EV was approved by the FDA under accelerated approval in December 2019 based on results from the EV-201 trial in 125 adult patients with la/mUC previously treated with platinum and anti-PD-1/ L1 therapy, which showed a confirmed objective response rate of $44 \%$ (95\% $\mathrm{CI}=35.1 \%-53.2 \%$ ) and a $\mathrm{CR}$ rate of $12 \%{ }^{18,65}$ Further study is needed on the number of patients who receive a subsequent therapy following progression on IO since the introduction of new therapies in this setting.

\section{LIMITATIONS}

The study reflects a period in time shortly after approvals for IOs and, as such, a limited number of studies were identified with data on IO and, in particular, post-IO treatment patterns. Differences in study design and data reporting made comparisons across studies and within and among countries difficult. LOT was not specified in many studies. Many of the identified studies included $<30$ patients or reported data that were $>5$ years old, and so may not be reflective of current treatment patterns. The majority of published studies were from the United States and Japan and cannot be considered representative of other countries. In particular, very limited data were available for Canada and Spain. Because no epidemiology studies meeting inclusion criteria were identified, epidemiology websites were used. The websites reported data for bladder cancer overall; epidemiology data on urothelial carcinoma specifically were not available.

\section{Conclusions}

Bladder cancer remains one of the most prevalent cancers worldwide with a significant number of new cases and deaths each year. Use of cisplatin, the recommended first-line treatment for patients with la/mUC, varies widely both within and among countries, indicating that many eligible patients are not receiving guidelinerecommended therapy. There has been an increase in the use of IO therapy for la/mUC in recent years, however, due to a lack of published real-world studies as well as the relative recency of these clinical practice changes, very little is known outside clinical trials. The few published real-world studies reported relatively high levels of treatment discontinuation in patients treated with anti-PD-1/L1 therapies, yet only a small percentage received subsequent therapy, indicating a high need for novel therapies that are safe and efficacious.

\section{DISCLOSURES}

This study was funded by Astellas/Seagen. Hepp is an employee of and owns stock in Seagen. Shah was a contractor for Astellas Pharma at the time of the study and owns stock in Pfizer. Smoyer is an employee and shareholder of Envision Pharma Group, paid consultants to Seagen. Vadagam was an employee of Envision Pharma Group, paid consultants to Seagen, at the time of the study.

Parts of these data have been presented at the International Society for Pharmacoeconomics and Outcomes Research (ISPOR) 2019 Annual Meeting; May 18-22, 2019; New Orleans, LA.

\section{ACKNOWLEDGMENTS}

Medical writing support was provided by Jonathon Carthy of Curo, a division of Envision Pharma Group, and funded by Astellas/Seagen.

\section{REFERENCES}

1. Ferlay J, Ervik M, Lam F, et al. Cancer today: data visualization tools for exploring the global cancer burden in 2018. 2018 Accessed November 25, 2020. https://gco. iarc.fr/today

2. Bamias A, Tzannis K, Harshman LC, et al. Impact of contemporary patterns of chemotherapy utilization on survival in patients with advanced cancer of the urinary tract: a Retrospective International Study of Invasive/Advanced Cancer of the Urothelium (RISC). Ann Oncol. 2018;29(2):361-69.

3. Fleshner NE, Herr HW, Stewart AK, Murphy GP, Mettlin C, Menck HR. The National Cancer Data Base report on bladder carcinoma: the American College of Surgeons Commission on Cancer and the American Cancer Society. Cancer. 1996;78(7):1505-13. 
4. Boustead GB, Fowler S, Swamy R, Kocklebergh R, Hounsome L; Section of Oncology-BAUS. Stage, grade and pathological characteristics of bladder cancer in the UK: British Association of Urological Surgeons (BAUS) urological tumour registry. BJU Int. 2014;113(6):924-30.

5. National Cancer Institute Surveillance, Epidemiology, and End Results Program. Cancer stat facts: bladder cancer. 2020. Accessed November 25, 2020. https://seer. cancer.gov/statfacts/html/urinb.html

6. von der Maase H, Sengelov L, Roberts JT, et al. Long-term survival results of a randomized trial comparing gemcitabine plus cisplatin, with methotrexate, vinblastine, doxorubicin, plus cisplatin in patients with bladder cancer. J Clin Oncol. 2005;23(21):4602-08.

7. Bellmunt J, Orsola A, Leow JJ, et al. Bladder cancer: ESMO Practice Guidelines for diagnosis, treatment and follow-up. Ann Oncol. 2014;25(Suppl 3):iii40-48.

8. Dash A, Galsky MD, Vickers AJ, et al. Impact of renal impairment on eligibility for adjuvant cisplatin-based chemotherapy in patients with urothelial carcinoma of the bladder. Cancer. 2006;107(3):506-13.

9. Galsky MD, Hahn NM, Rosenberg J, et al. Treatment of patients with metastatic urothelial cancer "unfit" for cisplatinbased chemotherapy. J Clin Oncol. 2011;29(17):2432-38.

10. De Santis M, Bellmunt J, Mead G, et al. Randomized phase II/III trial assessing gemcitabine/carboplatin and methotrexate/carboplatin/vinblastine in patients with advanced urothelial cancer "unfit" for cisplatin-based chemotherapy: phase II-results of EORTC study 30986. J Clin Oncol. 2009;27(33):5634-39.

11. National Cancer Institute. FDA approves immunotherapy drugs for patients with bladder cancer. May 30, 2017. Accessed November 25, 2020. https://www.cancer.gov/newsevents/cancer-currents-blog/2017/ approvals-fda-checkpoint-bladder
12. National Comprehensive Cancer Network. NCCN clinical practice guidelines V.5.2020. 2020. Accessed November 25, 2020. nccn.org/professionals/physician gls/default.aspx

13. Flaig TW, Spiess PE, Agarwal N, et al. NCCN guidelines insights: bladder cancer, version 5.2018. J Natl Compr Canc Netw. 2018;16(9):1041-53.

14. European Society for Medical Oncology. eUpdate: bladder cancer treatment recommendations. December 16, 2019.

Accessed November 25, 2020. https:// www.esmo.org/guidelines/genitourinarycancers/bladder-cancer/eupdate-bladder-cancer-treatment-recommendations3

15. Rosenberg JE, Hoffman-Censits J, Powles T, et al. Atezolizumab in patients with locally advanced and metastatic urothelial carcinoma who have progressed following treatment with platinum-based chemotherapy: a singlearm, multicentre, phase 2 trial. Lancet. 2016;387(10031):1909-20.

16. Bellmunt J, de Wit R, Vaughn DJ, et al. Pembrolizumab as second-line therapy for advanced urothelial carcinoma. N Engl J Med. 2017;376(11):1015-26.

17. Powles T, Durán I, van der Heijden MS, et al. Atezolizumab versus chemotherapy in patients with platinum-treated locally advanced or metastatic urothelial carcinoma (IMvigor211): a multicentre, open-label, phase 3 randomised controlled trial. Lancet. 2018;391(10122):748-57.

18. U.S. Food and Drug Administration. FDA grants accelerated approval to enfortumab vedotin-ejfv for metastatic urothelial cancer. December 18, 2019. Accessed November 25, 2020. https:// www.fda.gov/drugs/resources-informa tion-approved-drugs/fda-grants-accelerated-approval-enfortumab-vedotin-ejfvmetastatic-urothelial-cancer

19. U.S. Food and Drug Administration. FDA grants accelerated approval to erdafitinib for metastatic urothelial carcinoma. April 12, 2019. Accessed November 25, 2020. https://www.fda.gov/drugs/ resources-information-approved-drugs/ fda-grants-accelerated-approval-erdafi$\underline{\text { tinib-metastatic-urothelial-carcinoma }}$
20. Alfred Witjes J, Lebret T, Compérat EM, et al. Updated 2016 EAU guidelines on muscle-invasive and metastatic bladder cancer. Eur Urol. 2017;71(3):462-75.

21. National Institute for Health and Care Excellence. Vinflunine for the treatment of advanced or metastatic transitional cell carcinoma of the urothelial tract: technology appraisal guidance (TA272). January 23, 2013. Accessed November 25, 2020. https://www.nice.org.uk/guidance/ ta272/chapter/1-Guidance

22. Cumberbatch MGK, Jubber I, Black PC, et al. Epidemiology of bladder cancer: a systematic review and contemporary update of risk factors in 2018. Eur Urol. 2018;74(6):784-95.

23. Wells GA, Shea B, O'Connell D, et al. The Newcastle-Ottawa Scale (NOS) for assessing the quality of nonrandomised studies in meta-analyses. 2019. Accessed November 25, 2020. http://www.ohri.ca/programs/ clinical epidemiology/oxford.asp

24. Niu Q, Lu Y, Wu Y, et al. The effect of marital status on the survival of patients with bladder urothelial carcinoma: a SEER database analysis. Medicine (Baltimore). 2018;97(29):e11378.

25. Bharmal M, Guenther S, Kearney M. Epidemiology of locally advanced or metastatic urothelial cancer in the US, Europe and Japan. Value Health. 2017;20(9):A419.

26. Matsuda T, Okuyama A. Incidence rate for bladder cancer in Japanese in Japan and in the United States from the Cancer Incidence in Five Continents. Jpn J Clin Oncol. 2017;47(3):284-85.

27. Andreassen BK, Aagnes B, Gislefoss R, Andreassen $M$, Wahlqvist R. Incidence and survival of urothelial carcinoma of the urinary bladder in Norway 1981-2014. BMC Cancer. 2016;16(1):799.

28. Badar F, Mahmood S, Yusuf MA, Sultan F. Epidemiology of cancers in Lahore, Pakistan, 2010-2012: a crosssectional study. BMJ Open. 2016;6(6):e011828.

29. Guillaume L, Guy L. Epidemiology of and risk factors for bladder cancer and for urothelial tumors [in French]. Rev Prat. 2014;64(10):1372-74, 78-80. 
30. Burger M, Catto JW, Dalbagni G, et al. Epidemiology and risk factors of urothelial bladder cancer. Eur Urol. 2013;63(2):234-41.

31. Hegele A, Goebell P, Matz U, Neuhaus T. Monotherapy with intravenous vinflunine in patients with advanced or metastatic urothelial cancer after failure of a platinum-containing regimen: a retrospective analysis of German routine data. Urol Int. 2014;92(2):174-79.

32. Morales-Barrera R, Suárez C, Valverde C, et al. Do patients with metastatic urothelial carcinoma benefit from docetaxel as second-line chemotherapy? Clin Transl Oncol. 2014;16(1):102-06.

33. Moriceau G, Vallard A, Rivoirard R, et al. Real-world vinflunine outcomes in bladder cancer in a single-institution study: moving beyond clinical trials. Clin Genitourin Cancer. 2015;13(6):588-92.

34. Schinzari G, Rossi E, Pierconti F, et al. Monoinstitutional real world experience in management of Vinflunine as second line therapy for transitional cell carcinoma of the urothelium. Oncotarget.

2018;9(9):8765-71.

35. Szabados B, van Dijk N, Tang YZ, et al. Response rate to chemotherapy after immune checkpoint inhibition in metastatic urothelial cancer. Eur Urol. 2018;73(2):149-52.

36. Kobayashi K, Matsuyama H, Shimizu K, et al. Clinical significance of a second-line chemotherapy regimen with paclitaxel, ifosfamide and nedaplatin for metastatic urothelial carcinoma after failure of cisplatin-based chemotherapy. Jpn J Clin Oncol. 2016;46(8):775-80.

37. Matsui Y, Nishiyama H, Yoshimura K, et al. The effect of gemcitabine/paclitaxel chemotherapy on the survival of patients with metastatic urothelial cancers. Int J Clin Oncol. 2013;18(2):321-28.

38. Yoneyama T, Tobisawa Y, Yoneyama T, et al. Carboplatin-based combination chemotherapy for elderly patients with advanced bladder cancer. Int J Clin Oncol. 2015;20(2):369-74.
39. Mitsuzuka K, Yamashita S, Namiki S, et al. Low-dose maintenance gemcitabinecarboplatin chemotherapy could be an alternative to continuous standard chemotherapy for patients with metastatic urothelial carcinoma. Int J Urol. 2014;21(11):1114-19.

40. Terakawa T, Miyake H, Yokoyama N, et al. Clinical outcome of paclitaxel and carboplatin as second-line chemotherapy for advanced urothelial carcinoma resistant to first-line therapy with gemcitabine and cisplatin. Urol Int. 2014;92(2):180-85.

41. Galsky MD, Pal SK, Lin SW, et al. Realworld effectiveness of chemotherapy in elderly patients with metastatic bladder cancer in the United States. Bladder Cancer. 2018;4(2):227-38.

42. Malangone-Monaco E, Wilson K, Varker H, et al. Title: a real-world study of chemotherapy treatment and costs in metastatic urothelial cancer (mUC) patients in the United States. J Clin Oncol. 2017;35(15 Suppl):e16009.

43. Necchi A, Mariani L, Vullo SL, et al. Impact of number of cycles of platinum-based first-line chemotherapy for advanced urothelial carcinoma. J Clin Oncol. 2018;36(6 Suppl):426.

44. Doshi GK, Bhanegaonkar A, Bharmal $\mathrm{M}$, et al. SPEAR-bladder (study informing treatment pathway decision in bladder cancer): first- through third-line time to treatment failure in the U.S. J Clin Oncol. 2018;36(15 Suppl):4544.

45. Kunthur A, Siegel ER, Govindarajan

R. Cisplatin and gemcitabine versus carboplatin and gemcitabine in metastatic bladder cancer: survival analysis of veterans' health care data. J Clin Oncol. 2017;35(15 Suppl):e16023.

46. Kikuchi E, Miyazaki J, Yuge K, et al. Do metastatic upper tract urothelial carcinoma and bladder carcinoma have similar clinical responses to systemic chemotherapy? A Japanese multi-institutional experience. Jpn J Clin Oncol. 2016;46(2):163-69.
47. Muto S, Abe H, Noguchi T, et al. Maintenance monotherapy with gemcitabine after standard platinumbased chemotherapy in patients with advanced urothelial cancer. Int J Urol. 2015;22(5):490-94.

48. Flannery K, Boyd M, Black-Shinn J, Robert N, Kamat AM. Outcomes in patients with metastatic bladder cancer in the USA: a retrospective electronic medical record study. Future Oncol. 2019;15(12):1323-34.

49. Simeone JC, Nordstrom BL, Patel K, Klein AB, Horne L. Real-world survival and treatment patterns of patients with locally advanced or metastatic urothelial carcinoma treated with second-line therapy after platinum-based chemotherapy. J Clin Oncol. 2018;36(6 Suppl):466.

50. Barata PC, Gopalakrishnan D, Koshkin VS, et al. Atezolizumab in metastatic urothelial carcinoma outside clinical trials: focus on efficacy, safety, and response to subsequent therapies. Target Oncol. 2018;13(3):353-61.

51. Sonpavde G, Pond GR, Mullane S, et al. Outcomes in patients with advanced urothelial carcinoma after discontinuation of programmed death (PD)-1 or PD ligand 1 inhibitor therapy. BJU Int. 2017;119(4):579-84.

52. Yip SM, Kaiser J, Li H, North S, Heng DYC, Alimohamed NS. Real-world outcomes in advanced urothelial cancer and the role of neutrophil to lymphocyte ratio. Clin Genitourin Cancer. 2018;16(3):e637-44.

53. Wei XX, Lundgren K, Teo MY, et al. First-line PD-1/PD-L1 inhibitor followed by carboplatin (carbo)-based chemotherapy (chemo) or the reverse sequence in cisplatin-ineligible metastatic urothelial cancer (mUC) patients (pts). J Clin Oncol. 2018;36(15 Suppl):e16517.

54. Vander Velde N, Guerin A, Ionescu-Ittu R, et al. Comparative effectiveness of non-cisplatin first-line therapies for metastatic urothelial carcinoma: phase 2 IMvigor210 study versus US patients treated in the Veterans Health Administration. Eur Urol Oncol. 2019;2(1):12-20. 
55. Niegisch G, Gerullis H, Lin SW, et al. A real-world data study to evaluate treatment patterns, clinical characteristics and survival outcomes for first- and secondline treatment in locally advanced and metastatic urothelial cancer patients in Germany. J Cancer. 2018;9(8):1337-48.

56. Passalacqua R, Lazzarelli S, Donini M, et al. Real-life clinical practice results with vinflunine in patients with relapsed platinum-treated metastatic urothelial carcinoma: an Italian multicenter study (MOVIE-GOIRC 01-2014). BMC Cancer. 2017;17(1):493.

57. Holmsten K, Dohn L, Jensen NV, et al. Vinflunine treatment in patients with metastatic urothelial cancer: a Nordic retrospective multicenter analysis. Oncol Lett. 2016;12(2):1293-300.

58. Hussain SA, Ansari J, Huddart R, et al. VICTOR: vinflunine in advanced metastatic transitional cell carcinoma of the urothelium: a retrospective analysis of the use of vinflunine in multi-centre real life setting as second line chemotherapy through Free of Charge Programme for patients in the UK and Ireland. Int J Oncol. 2017;50(3):768-72.

59. European Association of Urology. Guidelines: muscle-invasive and metastatic bladder cancer. 2020. Accessed November 25, 2020. https://uroweb.org/ guideline/bladder-cancer-muscle-invasive-and-metastatic/

60. Ghatalia P, Zibelman M, Geynisman DM, Plimack E. Approved checkpoint inhibitors in bladder cancer: which drug should be used when? Ther Adv Med Oncol. 2018;10:1758835918788310.

61. Sylvester RJ, van der Meijden AP, Oosterlinck W, et al. Predicting recurrence and progression in individual patients with stage Ta T1 bladder cancer using EORTC risk tables: a combined analysis of 2596 patients from seven EORTC trials. Eur Urol. 2006;49(3):466-65.

62. Parikh RB, Feld EK, Galsky MD, et al. First-line immune checkpoint inhibitor use in cisplatin-eligible patients with advanced urothelial carcinoma: a secular trend analysis. Future Oncol. 2020;16(2):4341-45.
63. Parikh RB, Adamson BJS, Khozin S, et al. Association between FDA label restriction and immunotherapy and chemotherapy use in bladder cancer. JAMA. 2019;322(12):1209-11.

64. Morgans AK, Rahma OE, Mhatre SK, et al. Treatment (tx) characteristics of patients (pts) with locally advanced or metastatic urothelial cancer (mUC) receiving checkpoint inhibitor (CPI) monotherapy in a U.S. clinical practice. J Clin Oncol. 2019;37(15 Suppl):4526.

65. Rosenberg JE, O’Donnell PH, Balar AV, et al. Pivotal trial of enfortumab vedotin in urothelial carcinoma after platinum and anti-programmed death 1 /programmed death ligand 1 therapy. J Clin Oncol. 2019;37(29):2592-600.

66. Locke JA, Pond GR, Sonpavde G, et al. Cisplatin- versus non-cisplatin-based first-line chemotherapy for advanced urothelial carcinoma previously treated with perioperative cisplatin. Clin Genitourin Cancer. 2016;14(4):331-40.

67. Flannery K, Black-Shinn J, Boyd M, Kamat AM. Real world treatment utilization and outcomes of patients with metastatic bladder cancer by cisplatin eligibility in U.S. community oncology practice. J Clin Oncol. 2017;35(15 Suppl):e16016.

68. Fisher M, Shenolikar R, Miller PJE, Walker MS, Fenton MJ. Treatment patterns and outcomes in metastatic bladder cancer in community oncology settings. J Clin Oncol. 2017;35(6 Suppl):396.

69. Zargar-Shoshtari K, Kongnyuy M, Sharma P, et al. Clinical role of additional adjuvant chemotherapy in patients with locally advanced urothelial carcinoma following neoadjuvant chemotherapy and cystectomy. World J Urol. 2016;34(11):1567-73.

70. Bambury RM, Benjamin DJ, Chaim JL, et al. The safety and efficacy of singleagent pemetrexed in platinum-resistant advanced urothelial carcinoma: a large single-institution experience. Oncologist. 2015;20(5):508-15.
71. Yelfimov DA, Frank I, Boorjian SA, Thapa P, Cheville JC, Tollefson MK. Adjuvant chemotherapy is associated with decreased mortality after radical cystectomy for locally advanced bladder cancer. World J Urol. 2014;32(6):1463-68.

72. Laurent M, Brureau L, Demery ME, et al. Early chemotherapy discontinuation and mortality in older patients with metastatic bladder cancer: the AGEVIM multicenter cohort study. Urol Oncol. 2017;35(1):34.e9-6.

73. Canadian Cancer Society. Bladder cancer statistics. 2020. Accessed November 25, 2020. http://www.cancer. ca/en/cancer-information/cancer-type/ bladder/statistics/?region=on

74. National Cancer Center Japan. Projected cancer statistics, 2018. September 15, 2018. Accessed November 25, 2020. https://ganjoho.jp/en/public/ statistics/short_pred.html

75. Cancer Research UK. Bladder cancer statistics. 2020. Accessed November 25, 2020. https://www.cancerresearchuk. org/health-professional/cancerstatistics/statistics-by-cancer-type/ bladder-cancer

76. European Cancer Information System. Data explorer: estimates of cancer incidence and mortality in 2018, for all countries. 2020. Accessed November 25, 2020. https://ecis.jrc.ec.europa.eu/ explorer.php

77. Kearney M, Esposito D, Russo L, et al. Locally advanced or metastatic urothelial carcinoma in the United States: economic burden and healthcare utilization. Value Health. 2018;21(Suppl 1):S45.

78. Simeone JC, Nordstrom BL, Patel K, Mann H, Klein AB, Horne L. Treatment patterns and overall survival in metastatic urothelial carcinoma in a real-world, U.S. setting. Cancer Epidemiol. 2019;60:121-27.

79. Parikh RB, Galsky MD, Baxi SS, et al. Real-world trends in first-line checkpoint inhibitor use (CPI) in advanced urothelial cell carcinoma (aUC). J Clin Oncol. 2019;37(7 Suppl):392. 